\title{
Elucidation of density profile of self-assembled sitosterol + oryzanol tubules with small-angle neutron scattering
}

\author{
Arjen Bot, ${ }^{* a}$ Elliot P. Gilbert, ${ }^{b}$ Wim G. Bouwman, ${ }^{c}$ Hassan Sawalha, ${ }^{d e}$ \\ Ruud den Adel, ${ }^{a}$ Vasil M. Garamus, ${ }^{f}$ Paul Venema, ${ }^{d}$ Erik van der \\ Linden $^{d}$ and Eckhard Flöter ${ }^{a g}$
}

\author{
Received 12th February 2012, Accepted 28th February 2012 \\ DOI: $10.1039 / \mathrm{c} 2 \mathrm{fd} 20020 \mathrm{a}$
}

\begin{abstract}
Small-angle neutron scattering (SANS) experiments have been performed on selfassembled tubules of sitosterol and oryzanol in triglyceride oils to investigate details of their structure. Alternative organic phases (deuterated and nondeuterated decane, limonene, castor oil and eugenol) were used to both vary the contrast with respect to the tubules and investigate the influence of solvent chemistry. The tubules were found to be composed of an inner and an outer shell containing the androsterol group of sitosterol or oryzanol and the ferulic acid moieties in the oryzanol molecule, respectively. While the inner shell has previously been detected in SAXS experiments, the outer shell was not discernible due to similar scattering length density with respect to the surrounding solvent for X-rays. By performing contrast variation SANS experiments, both for the solvent and structurant, a far more detailed description of the self-assembled system is obtainable. A model is introduced to fit the SANS data; we find that the dimensions of the inner shell agree quantitatively with the analysis performed in earlier SAXS data (radius of $39.4 \pm 5.6 \AA$ for core and inner shell together, wall thickness of $15.1 \pm 5.5 \AA$ ). However, the newly revealed outer shell was found to be thinner than the inner shell (wall thickness $8.0 \pm 6.5 \AA$ ). The changes in the scattering patterns may be explained in terms of the contrast between the structurant and the organic phase and does not require any subtle indirect effects caused by the presence of water, other than water promoting the formation of sitosterol monohydrate in emulsions with aqueous phases with high water activity.
\end{abstract}

\footnotetext{
${ }^{a}$ Unilever Research and Development Vlaardingen, Olivier van Noortlaan 120, NL-3133 AT Vlaardingen, The Netherlands.E-mail: arjen.bot@unilever.com

${ }^{b}$ Bragg Institute, Australian Nuclear Science and Technology Organisation, Locked Bag 2001, Kirrawee DC, NSW 2232, Australia

'Department of Radiation, Radionuclides \& Reactors, Faculty of Applied Sciences, Delft University of Technology, Mekelweg 15, NL-2629 JB Delft, The Netherlands

${ }^{d}$ Laboratory of Physics and Physical Chemistry of Foods, Department of Agrotechnology and Food Sciences, Wageningen University, Bomenweg 2, NL-6703 HD Wageningen, The Netherlands

${ }^{e}$ Chemical Engineering and Material Science, An-Najah National University, P.O. Box 7, Nablus, Palestine

${ }^{f}$ Helmholtz-Zentrum Geesthacht: Zentrum für Material- und Küstenforschung, Max Planck Strasse 1, D-21502 Geesthacht, Germany

${ }^{g}$ Food Process Engineering, Department of Food Technology and Food Chemistry, Technical University Berlin, Königin Luise Strasse 22, D-14195 Berlin, Germany
} 


\section{Introduction}

In recent years, $\gamma$-oryzanol has been found to self-assemble with a range of plant sterols to form helical ribbons in triglyceride oil. ${ }^{1}$ The helical ribbon tubules can aggregate subsequently into a firm 'organogel' network. ${ }^{2}$ Most detailed work has been performed on the mixture of oryzanol with sitosterol, but similar assemblies are formed by oryzanol with the plant sterols ergosterol, stigmasterol, cholesterol and cholestanol. Small-angle X-ray scattering (SAXS) has been used to determine the tubule diameter and wall thickness, which were found to vary between 67 and $80 \AA$ A (diameter) and between 8 and $12 \AA$ (wall thickness), respectively, depending on the particular plant sterol used. ${ }^{3}$ The tubule is far in excess of $1000 \AA$ in length.

The oryzanol + plant sterol mixtures are part of a wider class of systems that have recently been investigated in order to reduce the reliance of the structuring of edible oils on crystalline fat (i.e. triglycerides rich in saturated fatty acids). ${ }^{4-8}$ Such nontriglyceride structuring systems for food-grade vegetable oils include fatty acids, fatty alcohols, monoglycerides (monoacylglycerols or MAGs), diglycerides (diacylglycerols or DAGs), phospholipids, ceramides, waxes, wax esters, or mixtures thereof ${ }^{9-13}$ and modified biopolymers. ${ }^{14}$ Some of these systems eliminate the relation between providing firmness to an oil-based system on the one hand and raising blood cholesterol when consumed in a food product on the other hand. This is a very desirable property since raised blood cholesterol levels are considered to be a risk factor for cardiovascular disease. ${ }^{4}$

The sensitivity of the sitosterol + oryzanol structurant to water was investigated recently, since most potential applications are foreseen in emulsion systems similar to margarine or butter. ${ }^{15}$ Generally, it was found that the firmness of water-in-oil (w/o) emulsion gels based on sitosterol + oryzanol mixtures is much lower than would be expected based on the organogel properties. ${ }^{16-18}$ This was explained by results from SAXS which demonstrated that water in emulsion gels binds to the sitosterol and promotes the formation of sitosterol monohydrate crystals. This prevents the self-assembly of sitosterol and oryzanol via hydrogen bonding. ${ }^{19}$ However, the detrimental effect of water on structuring by sitosterol and oryzanol can be prevented or slowed down in two ways: ${ }^{18}$ (i) reduction of the water activity below $\sim 0.9$, e.g. through the addition of $\mathrm{NaCl}$ or sugar to the water phase to make the formation of the monohydrate crystals energetically unfavourable; or (ii) changing the organic phase to a lower polarity oil (e.g. decane, limonene) with a water solubility below $\sim 0.1 \%$ to reduce the rate of monohydrate crystal formation.

One of the puzzling aspects of the SAXS scattering pattern in emulsions is the splitting of the first broad peak into a, possible, overlapping double peak, as is illustrated in Fig. 1 for emulsions with high water activity (i.e. no salt). For the remainder, both the position and width of this double peak are very similar to the corresponding parameters for the first peak for emulsions with low water activity (Fig. 1) or, indeed, for organogels, which makes it likely that the structure of the tubules in emulsions is very similar to that of the tubules in the organogels. The emulsion data with the double peak in SAXS cannot be fitted to a hollow tube model as found for the pure organogel data. ${ }^{17}$ Previously, however, it was found that the double peak can be constructed (qualitatively) using a combination of two hollow tubes, one with $\sim 70$ and one with $\sim 100 \AA$ diameter, with an essentially constant relative amplitude of both contributions to the SAXS data. ${ }^{17}$ The constant relative intensity of both parts of the double peak, suggests that these are aspects of a single feature: a more complex wall structure than a tubule with one wall of finite thickness. One of the more simple explanations is that the electron density of the tubule is not completely homogeneous. One possible cause is the presence of water, since the latter may interfere with the formation of a hydrogen bonds between sitosterol and oryzanol. Alternatively, the different densities of stacked androsterol groups (or sterane cores) and protruding ferulic acid moieties of the oryzanol molecules may cause a contrast difference in the tubule wall. Unfortunately, neither hypothesis 

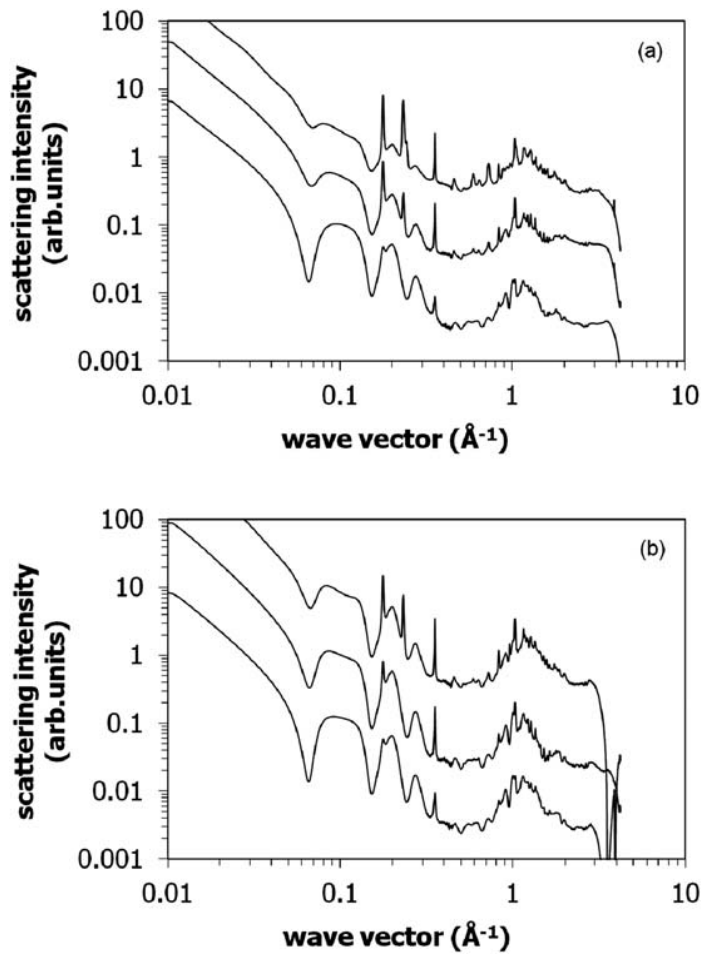

Fig. 1 SAXS for w/o emulsion gels in sunflower oil-(non-deuterated) decane mixtures, with $32 \%$ (40:60 sitosterol : oryzanol) as a structurant and 10\% aqueous phase at a temperature of $20{ }^{\circ} \mathrm{C}$ : (a) $90: 10$; (b) $80: 20$. The aqueous phase varies in each graph from top to bottom 0,10 and $20 \% \mathrm{NaCl}$. The scattering patterns have been shifted vertically for clarity.

can be tested easily in SAXS experiments since there is no way to independently vary the electron density of solvent and structurant.

As an alternative, small-angle neutron scattering (SANS) can be considered as an approach to obtain more detailed information at the nanometre length scale. ${ }^{20,21}$ SANS provides more freedom to choose the contrast, mainly by varying the degree of deuteration of solvent and structurant. The present study, therefore, will investigate the structure of the sitosterol + oryzanol structurant mixture in more detail using solvent contrast variation enabling selected phases to be highlighted. Amongst the parameters under study are the effects of water activity $(\mathrm{NaCl})$ and the effect of changing the oil in the organic phase.

\section{Experimental}

\section{Preparation of the emulsions}

In the present study, $\gamma$-oryzanol (Tsuno Rice Fine Chemicals, Wakayama, Japan) and tall oil sterol (78.5\% $\beta$-sitosterol, 10.3\% $\beta$-sitostanol, $8.7 \%$ campesterol and $2.5 \%$ of other minor sterols, Unilever, the Netherlands) were used as structurants. Sunflower oil (SF) (Reddy, NV Vandemoortele, Breda, the Netherlands), nondeuterated decane (>99\%, Sigma-Aldrich, The Netherlands), eugenol (99\%, Aldrich, The Netherlands), castor oil (Sigma, The Netherlands) and limonene (97\%, SigmaAldrich, The Netherlands) were used as solvents. Sodium chloride, $\mathrm{NaCl}$ (purity $>99 \%$, Merck, the Netherlands) or sucrose (>95\% (GC), Sigma, The Netherlands) dissolved in Milli-Q water solutions were used as the aqueous phase in the (w/o) 
emulsion. Deuterated decane was supplied by Acros Organics with D-enrichment $>98.5 \%$. Deuterated water $\left(\mathrm{D}_{2} \mathrm{O}\right)$ was supplied by Merck. All materials were used as received.

The organic phase was prepared by dissolving the structurants in the solvent at elevated temperatures $\left(\sim 100^{\circ} \mathrm{C}\right)$. The structurant concentration in the organic phase was kept constant at $32 \%(\mathrm{w} / \mathrm{w})$ with a fixed $\gamma$-oryzanol to $\beta$-sitosterol ratio of $60: 40 \% \mathrm{w} / \mathrm{w}$ (note that a relatively high structurant concentration was chosen to provide a clear scattering signal from the tubules. As such, organogels can be formed at much lower concentrations ${ }^{2}$ ). Aqueous solutions of different $\mathrm{NaCl}$ concentrations $(0,10,20 \% \mathrm{w} / \mathrm{w})$ were prepared and heated to $90{ }^{\circ} \mathrm{C}$. All percentages in formulations are expressed on a weight basis. To prepare the emulsion, the two phases were mixed at a fixed weight fraction of the aqueous phase $(10 \% \mathrm{w} / \mathrm{w})$ in a closed container at 90 ${ }^{\circ} \mathrm{C}$ and stirred at $1300 \mathrm{rpm}$ for $\sim 2 \mathrm{~min}$ using a magnetic stirrer. The resulting w/o emulsion was cooled down to room temperature and stirred until gelled. The solidified emulsion was subsequently stored at $5^{\circ} \mathrm{C}$ for 1 week before characterization. It should be noted that no additional emulsifiers or surfactants were added during emulsification and that the final w/o emulsion was stabilized by solidification.

\section{Small-angle neutron scattering (SANS)}

Preliminary SANS experiments were performed at the SANS-1 beamline at the Helmholtz-Zentrum Geesthacht in Geesthacht, Germany. ${ }^{22}$ A wavelength of $\lambda=$ $8.1 \AA$ was used and a full width at half maximum of $0.81 \AA$. Several sample-todetector configurations were used to cover the wave vector range $0.005<q / \AA^{-1}<$ 0.25 (sample-detector distance (SDD) from 0.7 to $9.7 \mathrm{~m}$ ) where $q=4 \pi \cdot \sin \theta / \lambda$ is the wave vector (and $2 \theta$ the scattering angle). The samples were contained in Hellma quartz cells of $2 \mathrm{~mm}$ path length. Samples were measured at $20^{\circ} \mathrm{C}$. The raw data were corrected for sample transmission and sample cell scattering by conventional procedures, azimuthally averaged, converted to an absolute scale and corrected for detector efficiency by dividing by the known incoherent scattering from $1 \mathrm{~mm}$ thick water. The scattering from solvents used for the sample preparation were subtracted as backgrounds. These experiments demonstrated that it was possible to obtain a clear tubule signature from sitosterol + oryzanol structured emulsion gels having deuterated decane as an organic phase by means of SANS (data not shown).

Final SANS experiments were performed on the $40 \mathrm{~m}$ Quokka instrument at the OPAL reactor (ANSTO, Sydney, Australia). ${ }^{23}$ A wavelength $\lambda=5.078 \AA$ and $14 \%$ wavelength resolution was used with source aperture diameter of $50 \mathrm{~mm}$. Three instrument configurations were used to cover the wave vector range $0.004<q / \AA^{-1}$ $<0.7(\mathrm{SDD}=20.10,3.20$, and $1.32 \mathrm{~m})$. The sample diameter used was selected by means of an automatic sample aperture changer within the instrument control system to maximise flux at the sample position but ensuring the scattering rate on the detector is within linearity in the detector response. Thus sample diameters of $15 \mathrm{~mm}, 12.5 \mathrm{~mm}$ and $7.5 \mathrm{~mm}$ were used at low, medium and high $q$ configurations respectively. Samples were contained in demountable cells of 1 or $2 \mathrm{~mm}$ thickness depending on the deuteration level of the samples and contained within a thermostatically controlled 20 -position automatic sample changer at $20{ }^{\circ} \mathrm{C}$.

SANS datasets were reduced, normalized and radially averaged using a package of macros in Igor (Wavemetrics, Lake Oswego, Oregon, USA) software originally written by Kline ${ }^{24}$ and modified to accept HDF5 data files from Quokka. Scattering curves are plotted as a function of absolute (SANS) intensity, I, versus $q$ using the attenuated direct beam of known attenuation coefficient. A model was used that will be introduced later in this paper (eqn (1)). Preliminary fitting established ranges for the free parameters: the core diameter was limited in the range between 15 and $30 \AA$, the wall thicknesses of inner and outer shell between 5 and $20 \AA$, the scattering length density of inner and outer shell between $5 \times 10^{-7}$ and $5 \times 10^{-6} \AA^{-2}$, the intensity scale factor was limited to positive values. The somewhat slanting background 
identified for emulsions containing deuterated decane introduced a further complicating factor to the fitting procedure. Since the outer shell fitting parameters were compensating for the background for some curves, the emulsions and organogels based on a pure deuterated decane organic phase were left out in the simultaneous fit. This exclusion has little effect on the fitting parameters.

Due to their firmness, small amounts of air were introduced in the samples, affecting the absolute intensity of the SANS scattering pattern. To correct for this, the scattering intensity was normalised to a linear combination of the solvents (water, organic phases) for each sample averaged over the wave vector range $0.6<$ $q / \mathrm{A}^{-1}<0.7$. This correction could amount to $25 \%$ of the (small) background contribution in some cases, but was usually smaller. Due to finite beam time allocation, only selected combinations of aqueous phase and organic phase systems could be measured.

\section{Small-angle X-ray scattering (SAXS)}

A number of supporting small-angle and wide-angle X-ray scattering (SAXS and WAXS, respectively) experiments were performed at the high-brilliance ID2 beamline of the European Synchrotron Radiation Facility (ESRF) in Grenoble, France. ${ }^{25}$ An incident X-ray wavelength of $\lambda=0.996 \AA$ was used. Two instrument configurations were used to cover the wave vector ranges $0.006<q / \AA^{-1}<0.45$ (SAXS) and $0.38<q / \AA^{-1}<3.78$ (WAXS) (SDD = 1.50 and $0.110 \mathrm{~m}$, respectively). The samples were $2.2 \mathrm{~mm}$ thick and held in an aluminium cell with thin mica windows. The sample temperature of $20{ }^{\circ} \mathrm{C}$ was controlled by Peltier elements.

The incident and transmitted X-ray beam intensities were recorded with each SAXS pattern and used to normalise the measured SAXS intensities. The normalized two-dimensional SAXS patterns were azimuthally averaged to obtain the scattered intensity as a function of $q$. The corresponding background intensity was subtracted and the resulting corrected scattered intensity presented here.

\section{Differential Scanning Calorimetry (DSC)}

DSC was performed using a Perkin Elmer Diamond DSC (Perkin-Elmer Co., Norwalk, CT). 7-15 mg sample was loaded in stainless steel pans, sealed and mounted in the DSC. Scans involved a temperature cycle from $0{ }^{\circ} \mathrm{C}$ to $120^{\circ} \mathrm{C}$ to $0{ }^{\circ} \mathrm{C}$ and back to $120{ }^{\circ} \mathrm{C}$ at a constant heating/cooling rate of $10^{\circ} \mathrm{C} \mathrm{min}$.

\section{Results}

\section{Solvent scattering}

While it would be desirable to use a deuterated analogue of sunflower oil as a solvent for conducting SANS studies, it is prohibitively expensive to acquire sufficiently large quantities. However, it has been shown that sitosterol + oryzanol tubules can be formed in a range of solvents. ${ }^{18}$ Amongst these, decane can be readily acquired commercially in a fully deuterated form. In these experiments, therefore, a range of solvents was investigated including deuterated decane as well as mixtures of sunflower oil with both non-deuterated and deuterated decane to enable selective contrast variation studies.

Data from non-deuterated decane, deuterated decane, sunflower oil, castor oil, eugenol and a number of sunflower oil : decane mixtures $(100: 0,90: 10,80: 20$ and $50: 50)$ are shown in Fig. 2. The SAXS and WAXS data show pronounced peaks (see Fig. 2a) with, for example, broad features at $0.3 \AA^{-1}$ and $1.5 \AA^{-1}$ for sunflower oil (cf ref. 1). The $0.3 \AA^{-1}$ 'long spacing' is attributed to the bilayer thickness in the melt, ${ }^{26}$ while the $1.5 \AA^{-1}$ 'short spacing' feature is generally attributed to intermolecular adjacently-packed fatty acid chains and intramolecular atom-atom 

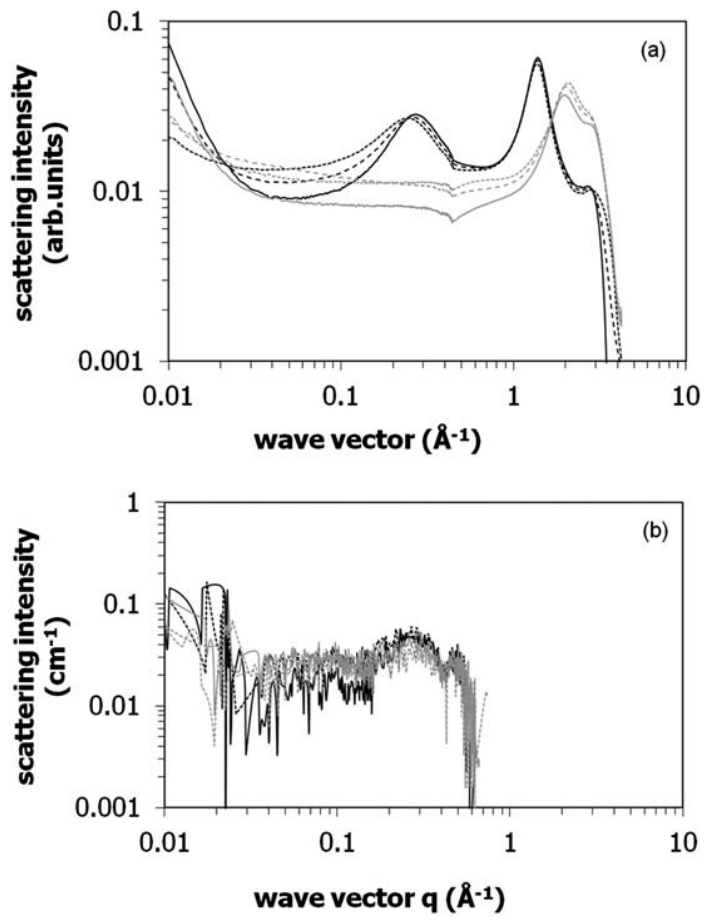

Fig. 2 Examples of (a) SAXS (and WAXS) and (b) SANS data for a selection of (non-deuterated) bulk phases. Black lines for sunflower oil : decane mixtures: (-) $100: 0 ;(---) 90: 10 ;(\cdots)$ 80 : 20; grey lines for water : salt mixtures: (-) $100: 0 ;(--) 90: 10 ;(\cdots) 80: 20$. The small cusp in the X-ray scattering data is caused by the transition between SAXS and WAXS detectors.

distances. The SANS data show only a weak feature at $0.3 \AA^{-1}$ (Fig. 2b). In part this is explained by the dominance of incoherent scattering.

\section{Organogels}

Fig. 3 shows SANS data for the organogels with various solvents. Fig. 3a shows the effect of the polarity of the solvents, (non-deuterated and deuterated) decane, sunflower oil, castor oil and eugenol (cf reference 18). The data clearly confirm tubule formation in non-deuterated and deuterated decane. Neutron scattering length densities (SLD) for the individual components are summarised in Table 1. The structure with deuterated decane is most pronounced which may be explained by the greater contrast between the solvent and the structurant.

Amongst the higher-polarity oils, the systems with sunflower and castor oil show very weak tubular patterns. The system with eugenol no longer exhibits evidence for tubules. Since these systems have also been studied by SAXS, which did reveal tubular patterns for all these samples, ${ }^{18}$ we attribute the absence of tubule scattering patterns to small contrast differences between structurant and solvent. Indeed, Table 1 indicates that the neutron scattering length densities for the solvents are all in the range $1.7 \times 10^{-7}$ to $1.3 \times 10^{-6} \AA^{-2}$, namely the same range as for sitosterol and oryzanol itself. In contrast, the neutron scattering length densities of nondeuterated and deuterated decane are outside this range, being $-4.9 \times 10^{-7}$ and $6.6 \times 10^{-6} \AA^{-2}$, respectively. This highlights the value of using deuterated labelling in these studies.

Fig. $3 \mathrm{~b}$ considers the more subtle effects of changes in solvent properties, in particular changes in the ratio between sunflower and decane, either deuterated or 

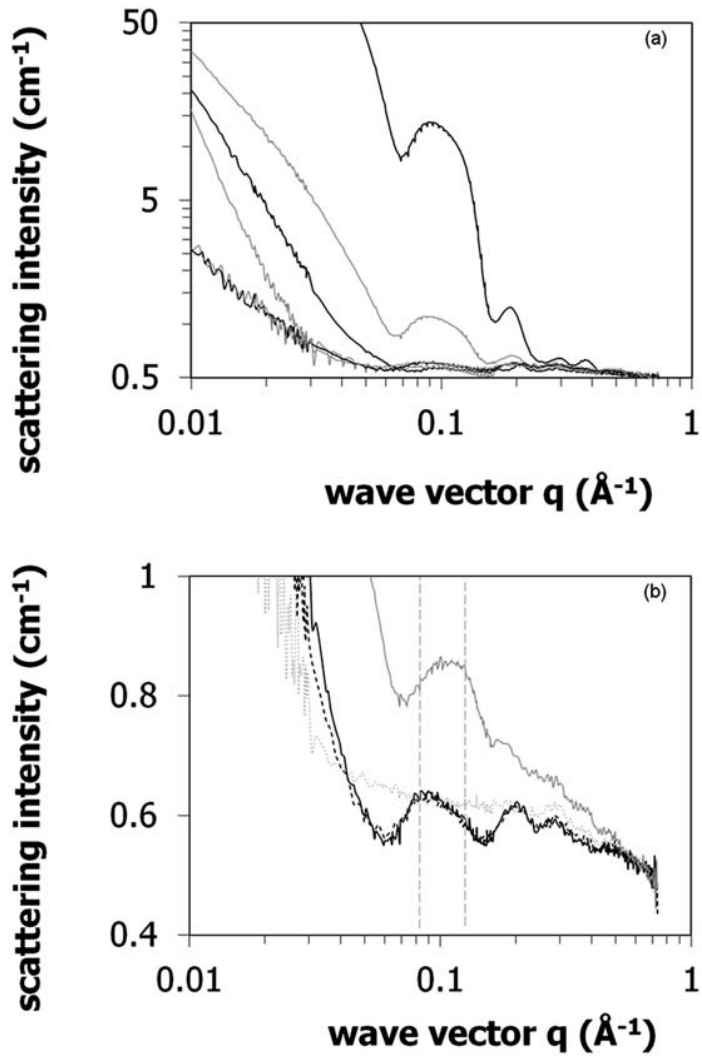

Fig. 3 SANS scattering patterns for organogels based on different organic phases: (a) deuterated decane, non-deuterated decane, eugenol, limonene, sunflower oil, castor oil (ordered from top to bottom according to intensity at small q); (b) various ratios of sunflower oil and decane (black curves are obtained from organogels containing non-deuterated decane, grey curves deuterated decane. The solid curves are obtained from organogels containing an $80: 20$ sunflower oil : decane organic phase, the dashed curves $90: 10)$. The vertical dashed lines indicate the position of the maxima of the first interference maximum for the organogels with deuterated or non-deuterated decane.

non-deuterated decane. The main variation in the scattering is influenced by the amount of deuterated decane in the organic phase. This is as expected, since changes in the ratio of non-deuterated decane and sunflower oil has little effect on the overall neutron scattering length density. Obviously, the substitution of sunflower oil for deuterated decane has a much greater effect on hydrogen density. An organic phase with $10 \%$ deuterated decane is rather well contrast-matched. However, tubules can be observed for the sample with $20 \%$ deuterated decane (low hydrogen density) or the samples without deuterated decane (high-hydrogen density). For the highhydrogen-density organic phase, the ratio of sunflower oil and decane did not affect the scattering pattern significantly.

It is interesting to note that the peak position of the first interference maximum (around $0.1 \AA^{-1}$ ) occurs at smaller wave vectors for high-hydrogen density organic phases (low neutron scattering length density, SLD) than for low-hydrogen density organic phases (high neutron SLD). Their positions at $2 \pi / q_{\mathrm{i}}=73$ and $53 \AA$, respectively, are indicated by the dashed vertical grey line. The most likely origin for the shift in peak position is in terms of an inhomogeneous neutron scattering length density distribution in the tubules, in particular a double walled tubule with a inner 
Table 1 Neutron scattering length densities (SLD) for components studied (http:// www.ncnr.nist.gov/resources/sldcalc.html)

\begin{tabular}{lll}
\hline Compound name & Compound formula & Neutron SLD $\left(\AA^{-2}\right)$ \\
\hline Decane (H) & $\mathrm{C}_{10} \mathrm{H}_{22}$ & $-4.88 \times 10^{-7}$ \\
Decane (D) & $\mathrm{C}_{10} \mathrm{D}_{22}$ & $6.58 \times 10^{-6}$ \\
Limonene & $\mathrm{C}_{10} \mathrm{H}_{16}$ & $2.47 \times 10^{-7}$ \\
Sunflower oil & $\left(\mathrm{C}_{57} \mathrm{H}_{101} \mathrm{O}_{6}\right)=\mathrm{C}_{3} \mathrm{H}_{5}\left(\mathrm{C}_{18} \mathrm{H}_{32} \mathrm{O}_{2}\right)_{3}$ & $2.25 \times 10^{-7}$ \\
Castor oil & $\left(\mathrm{C}_{57} \mathrm{H}_{107} \mathrm{O}_{9}\right)=\mathrm{C}_{3} \mathrm{H}_{5}\left(\mathrm{C}_{18} \mathrm{H}_{34} \mathrm{O}_{3}\right)_{3}$ & $1.91 \times 10^{-7}$ \\
Eugenol & $\mathrm{C}_{10} \mathrm{H}_{12} \mathrm{O}_{2}$ & $1.29 \times 10^{-6}$ \\
Water (H) & $\mathrm{H}_{2} \mathrm{O}$ & $-5.60 \times 10^{-7}$ \\
Water (D) & $\mathrm{D}_{2} \mathrm{O}$ & $6.48 \times 10^{-6}$ \\
Oryzanol & $\mathrm{C}_{40} \mathrm{H}_{58} \mathrm{O}_{4}$ & $7.21 \times 10^{-7}$ \\
Sitosterol & $\mathrm{C}_{29} \mathrm{H}_{50} \mathrm{O}$ & $1.68 \times 10^{-7}$ \\
Salt & $\mathrm{NaCl}$ &
\end{tabular}

high-hydrogen density (low SLD) cylinder and an outer low hydrogen density (high SLD) cylinder. This qualitative representation of the tubule structure will be developed in more detail in the Discussion section.

\section{Emulsions}

Emulsions with different organic phases. The next step is to introduce water in these systems by creating emulsions. Data obtained from corresponding emulsions are shown in Fig. 4. Again, it is clear that tubules are formed both in non-deuterated and deuterated decane emulsions. The tubule interference pattern is most pronounced in the emulsion based on deuterated decane. This is in line with the observation for the organogels (Fig. 3), and in agreement with the greater contrast difference between the solvent and the structurant (Table 1). Furthermore, tubules are still formed in limonene, although the limited contrast makes the scattering pattern less pronounced. Emulsions containing castor oil or SF (with pure water in the aqueous phase) show sharp crystallographic peaks instead of the characteristic broad tubule features. ${ }^{17,18}$ Peaks can be found at $d=2 \pi / q_{\mathrm{i}}=35$ and $27 \AA$ which

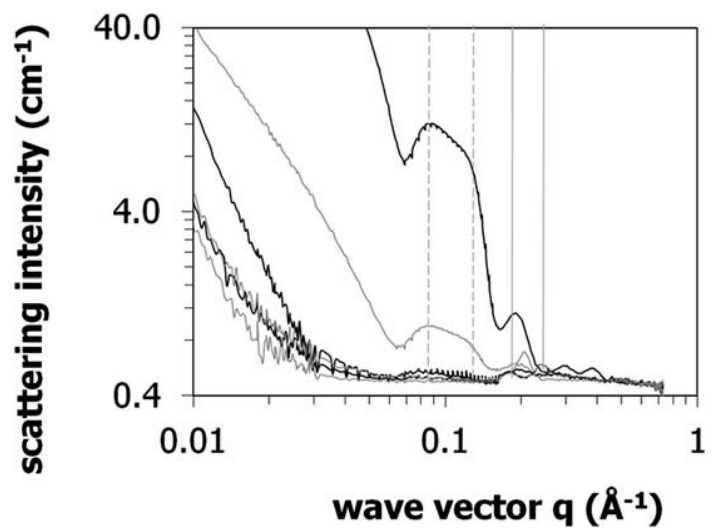

Fig. 4 SANS data for emulsions containing 10\% dispersed water and an organic phase based on various solvents: non-deuterated decane, deuterated decane, limonene, castor oil, sunflower oil, eugenol oil (ordered from top to bottom according to intensity at small $q$ ). The vertical grey lines indicate the position of the sitosterol monohydrate peaks (solid lines) and of the peaks identified in Fig. 3b (dashed lines). 
reflect the bilayer structure of sitosterol monohydrate. ${ }^{19,27,28}$ The positions of these peaks are indicated by the two grey solid vertical lines, for later comparison. Sitosterol in an eugenol-based emulsion shows a different crystalline reflection at $d=2 \pi / q_{\mathrm{i}}=\sim 31 \AA$ A, indicating a different type of organisation for the sitosterol monohydrate crystals in the more polar organic solvent. The two grey dashed vertical lines reflect the position of the peak maxima in the organogels (cf Fig. 3) and it can be concluded that these positions coincide with the maxima or shoulders in the SANS pattern for these emulsion gels.

Emulsions with different organic phases and different water activity. In previous SAXS experiments, emulsions based on decane were found to form tubules, whereas emulsions based on sunflower oil, castor oil or eugenol did not form stable tubules in the presence of a pure aqueous phase. ${ }^{18}$ This difference was attributed to the ability to form sitosterol hydrates if either the transport of water through the organic phase is fast (kinetic argument) or if the water activity of the emulsion is too high (equilibrium argument). This leaves two different routes to avoid hydrate formation and thus the intriguing possibility to study systems in which the contrast between structurant and organic phase is sufficient high to obtain the SANS signature of the tubules but, in addition, in which the water activity of the aqueous phase can be tuned to control the hydration behaviour of the sterols.

The DSC results shown in Fig. 5 demonstrate that sitosterol monohydrate formation occurs for up to $\sim 20 \%$ decane in sunflower oil. Although this unfortunately implies a very modest contrast between structurant and organic phase, the experiments in the previous section have indicated that the contrast is sufficient to still extract valuable information from such experiments. By creating an emulsion with an organic phase that has sufficient water solubility to still allow hydrate formation but that can be stabilised by tuning the water activity of the aqueous phase, it should be possible to study the effect of water on the structure of the sitosterol + oryzanol tubules, in particular the 'double first peak' pattern that is observed in many emulsions. ${ }^{17}$

SANS data from emulsions prepared from sunflower oil/deuterated decane mixtures with 10 and $20 \%$ deuterated decane, respectively, are shown in Fig. 6. For each organic phase, three salt levels were assessed $(0,10$ and $20 \% \mathrm{NaCl})$. Sharp crystallographic sitosterol monohydrate peaks are observed that were encountered previously for the salt-free emulsions with sunflower oil. ${ }^{18,19}$ Their position is marked by solid grey vertical lines. These reflections are much stronger in emulsions with $\mathrm{D}_{2} \mathrm{O}$ than in emulsions with $\mathrm{H}_{2} \mathrm{O}$ which reflects the enhanced contrast of the deuterated monohydrate with the hydrogenated organic phase. Emulsions with lower water activity due to the presence of salt (10 or $20 \%$ ) essentially do not exhibit

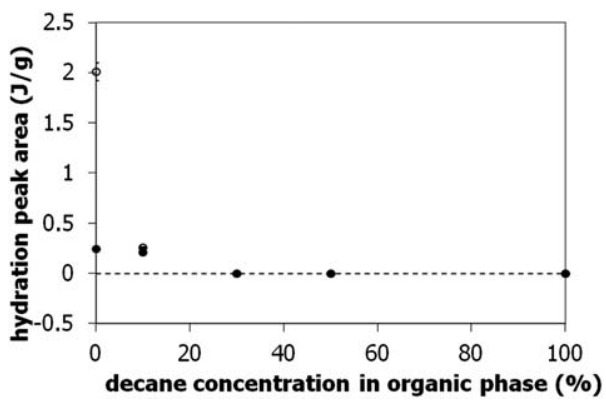

Fig. 5 Effect of (non-deuterated) decane : sunflower oil ratio on the area under the DSC hydration peak for a w/o emulsion with $10 \%$ pure water and $32 \% 40: 60$ sitosterol : oryzanol mixture on the organic phase: $(\bigcirc)$ first heating; $(\bigcirc)$ second heating. The data indicate that the transition vanishes at a decane concentration in sunflower oil of $20 \pm 10 \%$. 

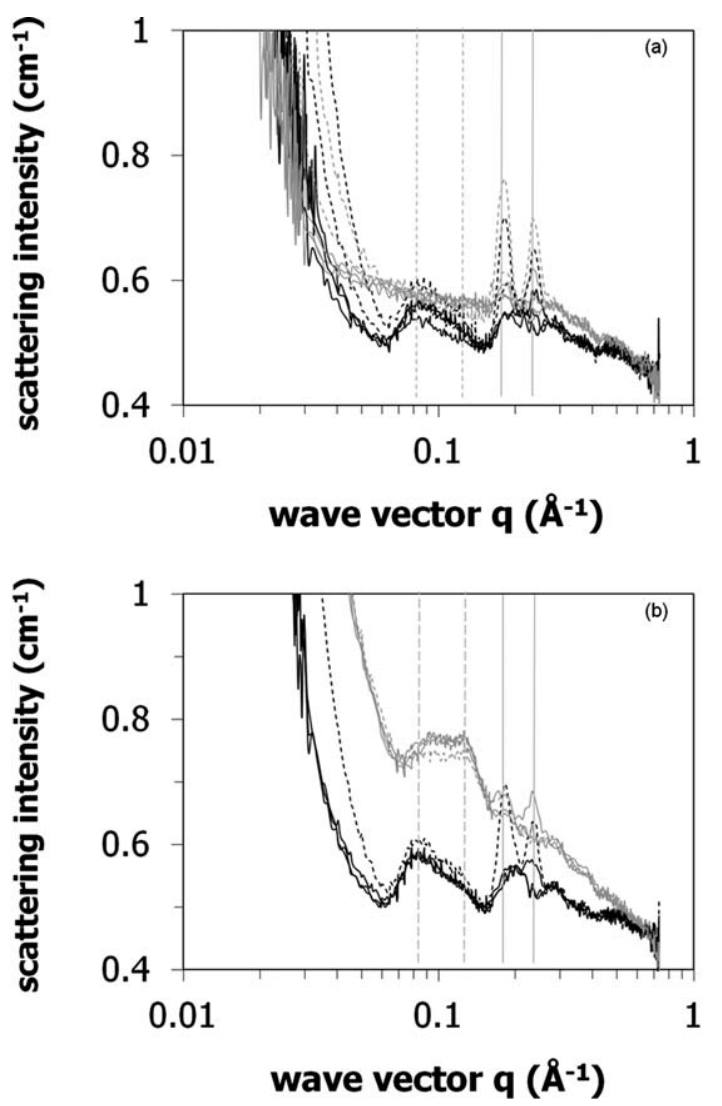

Fig. 6 Emulsions with sunflower oil : decane mixtures as the organic phase: (a) $90: 10$; (b) $80: 20$. The black curves are obtained from emulsions containing non-deuterated decane, the grey curves deuterated decane. The solid curves are obtained from emulsions containing $\mathrm{H}_{2} \mathrm{O}$, the dashed curves $\mathrm{D}_{2} \mathrm{O}$. In addition, the aqueous phase may contain $0,10,20 \% \mathrm{NaCl}$ but this is only reflected in sitosterol monohydrate formation ( $c f$. Fig. 1). The vertical grey lines indicate the position of the sitosterol monohydrate peaks (solid lines) and of the peaks identified in Fig. 3b (dashed lines).

sitosterol monohydrate formation with the scattering being dominated by the presence of tubules.

Furthermore, it can be seen that the high $q$ scattering for emulsions with deuterated decane does not seem to be completely flat, but decreases with increasing wave vector. In contrast, the pure deuterated decane sample does not show this effect indicating that selective deuteration enables a smaller-scale structure to be discerned. The emulsion with $10 \%$ deuterated decane in the organic phase appears to be almost contrast-matched with any potential tubules in the emulsion (Fig. 6a) - neutron SLDs are $0.500 \times 10^{-6} \AA^{-1}$ and $0.219 \times 10^{-6} \AA^{-1}$ for the sunflower oil : decane mixture and $40: 60$ sitosterol : oryzanol, respectively.

Fig. 6 confirms the observation in Fig. $3 b$ that the peak position of the first interference maximum at $\sim 0.1 \AA^{-1}$ occurs at smaller $q$ values for the emulsion with $20 \%$ non-deuterated decane compared to the emulsion with $20 \%$ deuterated decane in the organic phase (at $2 \pi / q_{\mathrm{i}}=73$ and $53 \AA$, respectively). Their positions are marked by dashed grey vertical lines. The effect implies, once more, the existence of two regions in the tubule wall: an inner wall with a high hydrogen density and an outer wall with a lower hydrogen density. The corresponding SAXS curves, using hydrogenated 
decane and water (Fig. 1), unequivocally demonstrate the presence of tubules in these emulsions, especially at lower water activities.

Except for the monohydrate peaks (position indicated by the solid vertical solid line), which become more pronounced at higher water activity, there is surprisingly little effect of the water on the SANS curves. The larger contrast in $\mathrm{D}_{2} \mathrm{O}$-containing emulsions increases the signal strength at small $q$ values, but does not change the peak shape of the first interference maximum at $\sim 0.1 \AA^{-1}$. This implies that if water affects the tubule shape directly, it can only involve very small amounts of water.

\section{Discussion}

One initial hypothesis in this work was that the double first interference maximum observed in SAXS was caused by a more complex wall structure of the tubules in emulsions, induced by the presence of water. It was anticipated that a comparison between samples that are identical except for the presence of either deuterated or non-deuterated water would mainly manifest itself by changes in the double-peaked scattering pattern around $0.1 \mathrm{~A}^{-1}$. This is not what is observed in the data, however. Changes in the 'water-contribution' to the scattering pattern always occur either in the range well beyond $0.1 \AA^{-1}$ and not close to the double-peaked interference maximum, and are associated with changes in the contrast of the sitosterol monohydrate peaks, or in the range well below $0.1 \AA^{-1}$.

It can be concluded, therefore, that the double maximum observed in SAXS for the first interference peak is not directly associated with water. In particular, both the organogel and the emulsion data indicate that the two peaks represent parts of the tubule wall with different hydrogen density, the inner part being denser than the outer part. A very natural interpretation of this observation is sketched in Fig. 7, in which the tubules are considered to be double-walled structures. The low-hydrogen density of the outer tubule is caused by the protruding ferulic acid moieties of the oryzanol in the partly deuterated organic phase; note that the latter are only present in about half of the molecules that form the tubule as sitosterol does not contain such a group. This model would confirm the inference that the ferulic acid moieties are located on the outside of the tubules, as has previously been proposed based on circumstantial evidence of aggregation behaviour at various sitosterol/oryzanol ratios ${ }^{4}$ and consideration of molecular stacking ${ }^{3}$.

Such a qualitative model can be turned into a somewhat more quantitative comparison by translating Fig. 7 in a scattering length density profile for the tubules to form cylinders with a core-shell-shell structure and whose length is much greater than the dimensions of the cross-section. The result is shown in Fig. 8. The associated fitting equation is:

$$
I(q)=\varphi \int_{0}^{\frac{\pi}{2}} f^{2}(q, \alpha) \cdot \sin \alpha d \alpha
$$

where $\varphi$ is the volume fraction of tubules and $\alpha$ is defined as the angle between the cylinder axis and the scattering vector, $q$. The integral over $\alpha$ averages the form factor over all possible orientations of the cylinder.

$$
\begin{gathered}
f(q, \alpha)=2 \cdot\left(\rho_{\text {solv }}-\rho_{1}\right) \cdot V_{\text {solv }} \cdot j_{0}(q(L / 2) \cdot \cos \alpha) \cdot J_{1}\left(q r_{\text {solv }} \cdot \sin \alpha\right) /\left(q r_{\text {solv }} \cdot \sin \alpha\right)+ \\
2 \cdot\left(\rho_{1}-\rho_{2}\right) \cdot V_{1} \cdot j_{0}\left(q\left((L / 2)+\left(r_{1}-r_{\text {solv }}\right)\right) \cdot \cos \alpha\right) \cdot J_{1}\left(q r_{1} \cdot \sin \alpha\right) /\left(q r_{1} \cdot \sin \alpha\right)+ \\
2 \cdot\left(\rho_{2}-\rho_{\text {solv }}\right) \cdot V_{2} \cdot j_{0}\left(q\left((L / 2)+\left(r_{2}-r_{\text {solv }}\right)\right) \cdot \cos \alpha\right) \cdot J_{1}\left(q r_{2} \cdot \sin \alpha\right) /\left(q r_{2} \cdot \sin \alpha\right)
\end{gathered}
$$

where the radii $\mathrm{r}_{i}$ are depicted in Fig. $8, j_{0}(x)=\sin (x) / x$, and $J_{1}(x)$ is the first order Bessel function and $\rho_{\mathrm{i}}$ the SLD of phase $i . L$ is the length of the cylindrical tubule. 


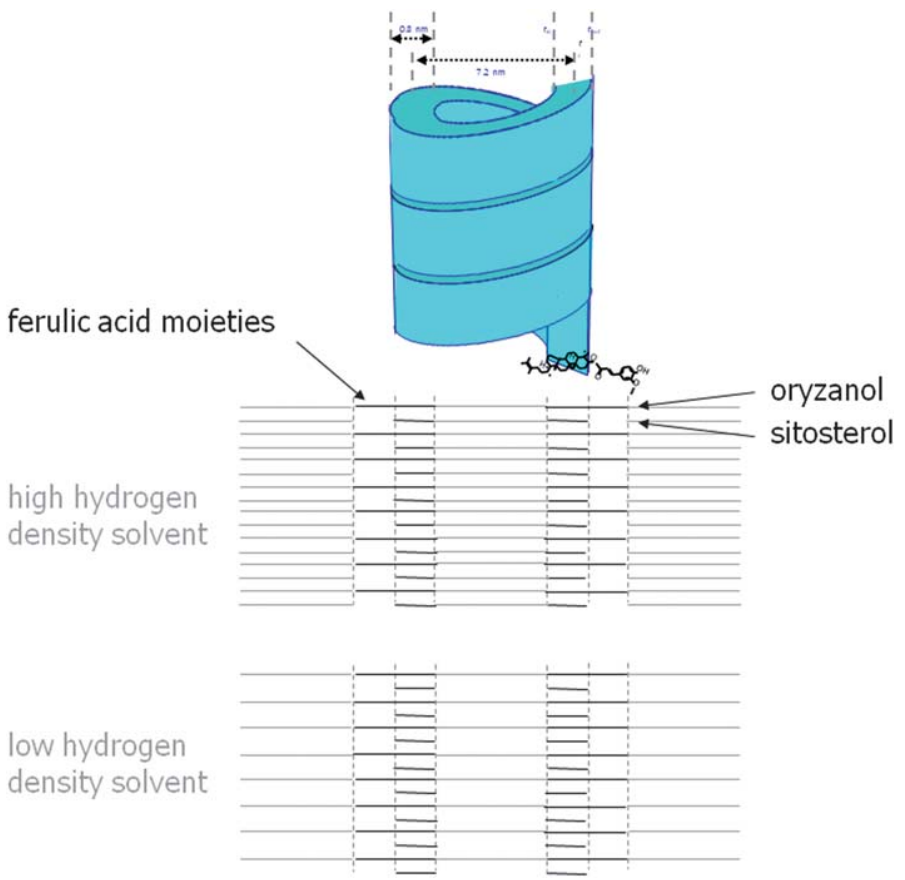

Fig. 7 Schematic representation of the high hydrogen density inside cylinder of the tubule (androsterol group in sterol and oryzanol) and the low hydrogen density outside cylinder (ferulic acid moieties of the oryzanol). The inner cylinder is revealed in a low hydrogen density solvent, and the outer cylinder in a high hydrogen density solvent.

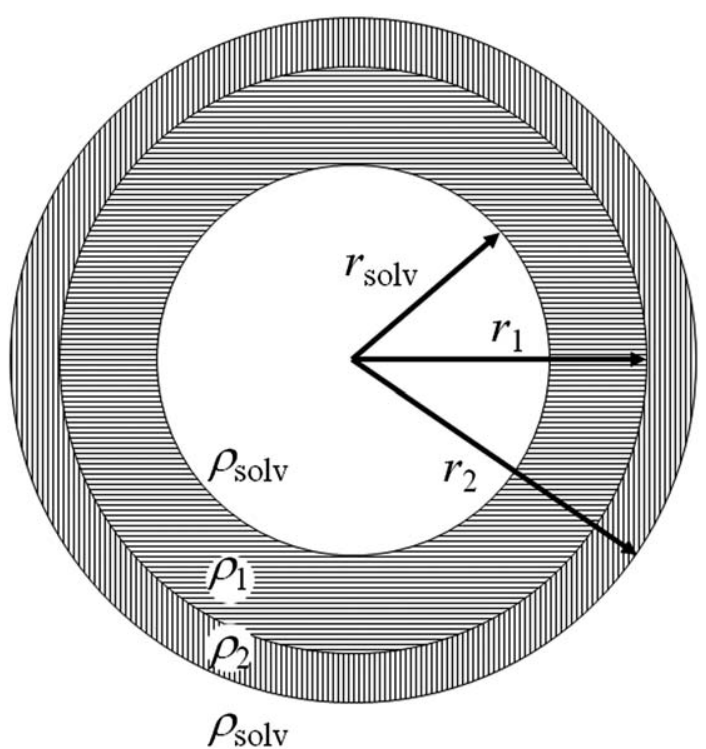

Fig. 8 Schematic representation of the double-walled tubule.

There are thus three regions defined: a core of radius, $r_{\text {solv }}$; an inner shell of thickness, $r_{1}-r_{\text {solv }}$ and an outer shell of thickness, $r_{2}-r_{1}$. The shell thickness is uniform over the entire tubule thus: 


$$
\begin{gathered}
V_{\text {solv }}=\pi r_{\text {solv }}^{2} L \\
V_{1}=\pi r_{1}^{2}\left(L+2 r_{1}\right) \\
V_{2}=\pi r_{2}^{2}\left(L+2 r_{1}+2 r_{2}\right)
\end{gathered}
$$

The equation reduces to the equation used for the analysis of previous SAXS experiments by taking $L \rightarrow \infty$ and $\rho_{1}=\rho_{2}$ for a hollow cylinder. ${ }^{29}$

Eqn (1) should still be considered as an oversimplification of the real system as it cannot perfectly generate the double first peak but will constitute enough of an advance over the existing model ${ }^{1,3}$ to warrant its use.

Eqn (1) was used to simultaneously fit all data for either the set of organogels or the set of emulsions over the $q$ range from 0.04 and $0.74 \AA^{-1}$ while fixing the neutron scattering length densities of the solvents (Table 1); this $q$ range contains the most relevant information concerning the tubule wall structure. For fitting the set of emulsions, the $q$ range featuring the sitosterol monohydrate peaks was excluded (range between 0.15 and $0.30 \AA^{-1}$ ). The data were used to extract the values for the diameter of the solvent-containing core and the wall thickness of the inner and outer shells. Note, therefore, that the model uses only six adjustable parameters, in addition to the scale factor and incoherent background of each curve. Some results are shown in Fig. 9.

Table 2 summarises the fitting parameters. The sum of core radius and inner shell agrees with the value that was obtained in earlier SAXS experiments: ${ }^{1,3} 39.4 \pm 5.6$ versus $39.1 \pm 2.2 \AA$ (note that the present result should be compared to $\mathrm{r}_{\text {out }}$ in reference [3], not to $r_{c}$ ). The thickness of the inner shell is consistent with the SAXS observation $(15.1 \pm 5.5$ vs. $8 \pm 2 \AA$ ); despite the enhanced signal to noise ratio in SAXS, it remains difficult to extract inner shell dimensions.

The data confirm that the masking of the outer shell in a SAXS experiment is a result of insufficient contrast. While the thickness of this outer shell cannot be determined precisely in the present SANS experiment, it seems to be thinner than

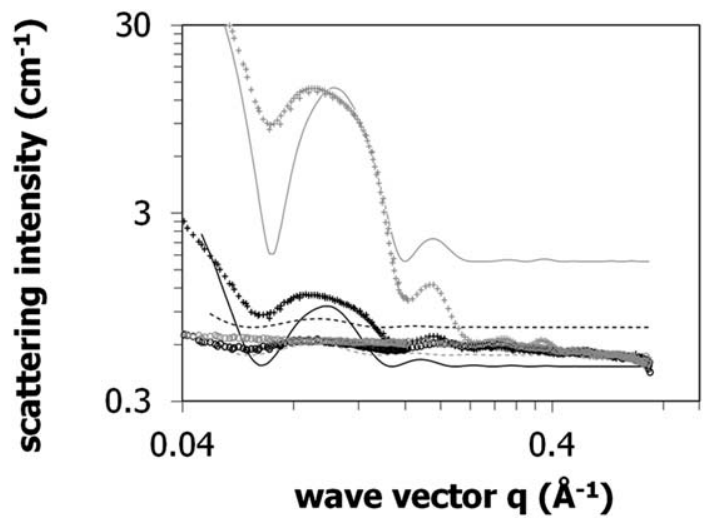

Fig. 9 Model fits (lines) to experimental data (symbols) on organogels using eqn (1). The black curves are obtained from organogels containing non-deuterated decane, the grey curves deuterated decane. The solid curves and $(+)$-symbols are obtained from emulsions containing pure decane as an organic phase, the dashed curves and (o)-symbols are obtained from emulsions containing $90: 10$ mixtures of sunflower oil : decane. 
Table 2 Fitting parameters for eqn (1)

\begin{tabular}{lll}
\hline Parameter & Value & St. dev \\
\hline Core radius, $r_{\text {solv }}(\AA)$ & 24.3 & 0.9 \\
Core length, $L(\AA)$ & $6.7 \times 10^{3}$ & $0.2 \times 10^{3}$ \\
Shell 1 thickness, $r_{1}-r_{\text {solv }}(\AA)$ & 15.1 & 5.5 \\
Shell 2 thickness, $r_{2}-r_{1}(\AA)$ & 8.0 & 6.5 \\
SLD shell $1, \rho_{1}\left(\AA^{-2}\right)$ & $7.8 \times 10^{-7}$ & $0.4 \times 10^{-7}$ \\
SLD shell $2, \rho_{2}\left(\AA^{-2}\right)$ & $1.3 \times 10^{-6}$ & $0.5 \times 10^{-6}$ \\
\hline
\end{tabular}

the inner shell: $8.0 \pm 6.5 \AA$. Finally, the length of the tubules is of the order of a few thousand $\AA$. For the present data, it suffices to note that the length of the tubules is much longer than their diameter.

Finally, the scattering length density of the inner and outer shell may provide clues whether their composition is in line with the hypothesis that the inner shell consists of androsterol groups (or sterane core) and the outer shell of ferulic acid moieties. Assuming a realistic density for the stacked molecules of approximately $1 \mathrm{~g} \mathrm{ml}^{-1}$, Table 3 indicates that the neutron scattering length density of the outer shell is very similar to that of ferulate and that of the inner shell is very close to androsterol.

Despite the success of the present analysis, it should be noted that the present model understates the true complexity of the structures formed. Indeed, the behaviour of the base line in the emulsions containing organic phases with higher deuterated decane concentration suggests more complex structures. It is anticipated that the present fit would be improved by explicitly considering alkyl groups protruding at the inside of the tubules into the organic phase (as Table 3 shows that the contrast difference between deuterated decane and the sitosterol alkyl chains is large) or by introducing a somewhat patchy distribution of ferulic acid moieties on the exterior surface of the tubules. The number of fitting parameters required would obviously increase as a result of the inclusion of pitch, roughness, polydispersity and other physically reasonable additions to the model, but might also explain the somewhat irregularly shaped first interference peak.

It would be of interest to conduct further contrast variation studies on these systems to elucidate more details of these self-assembled systems. One approach would be to focus on contrast differences but ignore any subtle effects due to the presence of water. Such an experiment would vary the contrast of the organic phase to the structurant without changing the physical properties (e.g. polarity) of the organic phase, for example by using mixtures of deuterated and non-deuterated decane. Hydration effects will not occur in such a system and they could be executed in organogel instead of emulsions. The second, more complex, approach would take hydration effects into account and would repeat the above experiment with mixtures of non-deuterated and deuterated triglycerides. These experiments would generate most additional information when performed on emulsions, but the first experiment could also be restricted to organogels. A deeper understanding of the structure of the tubules might also help to explain the kinetics of their formation. ${ }^{30,31}$

Table 3 Neutron scattering length densities (SLD) for a number of moieties in the sitosterol and oryzanol molecules (http://www.ncnr.nist.gov/resources/sldcalc.html)

\begin{tabular}{lll}
\hline Compound name & Compound formula & Neutron SLD $\left(\AA^{-2}\right)$ \\
\hline Androsterol & $\mathrm{C}_{19} \mathrm{H}_{30} \mathrm{O}$ & $4.37 \times 10^{-7}$ \\
Ferulate (minus one $\mathrm{O})$ & $\mathrm{C}_{10} \mathrm{H}_{9} \mathrm{O}_{3}$ & $1.71 \times 10^{-6}$ \\
Alkyl chain of sitosterol & $\mathrm{C}_{10} \mathrm{H}_{18}$ & $-3.67 \times 10^{-8}$ \\
\hline
\end{tabular}




\section{Conclusions}

The present neutron scattering experiments addressed the structure of self-assembled tubules composed of sitosterol and oryzanol that form in edible oils. By variation of the contrast between organic phase and structurant, it was possible to reveal that the cylindrical tubules are composed of an inner and an outer shell; this greatly extends our knowledge of the system which was based until now mostly on SAXS data. A core-shell-shell scattering model is necessary to describe the SANS with the scattering length densities consistent with an inner core consisting of the androsterol group in sitosterol and oryzanol for the inner shell, and with an outer shell composed of ferulic acid moieties (i.e. the ester part of oryzanol). The model indicates a core radius of $24.3 \pm 0.9 \AA$, and a wall thickness of inner and outer shell of $15.1 \pm$ $5.5 \AA$ and of $8.0 \pm 6.5 \mathrm{~A}$, respectively.

It was somewhat surprising to note that none of these changes were directly associated with the presence of water in organogels or emulsions, as had been anticipated. Instead, it seems that the more complex scattering patterns observed in SANS and in some cases in SAXS (especially for emulsions) should all be explained by changes in the contrast between organic phase and structurant.

\section{Acknowledgements}

The authors would like to thank T. Narayanan, M. Sztucki, S. Callow, J. Gummel and M. Fernandez Martinez (ID2 beam line, ESRF Grenoble, France) for their support.

\section{References}

1 A. Bot, R. den Adel and E. C. Roijers, J. Am. Oil Chem. Soc., 2008, 85, 1127-1134.

2 A. Bot and W. G. M. Agterof, J. Am. Oil Chem. Soc., 2006, 83, 513-521.

3 A. Bot, R. den Adel, E. C. Roijers and C. Regkos, Food Biophys., 2009, 4, 266-272.

4 M. Pernetti, K. F. van Malssen, E. Flöter and A. Bot, Curr. Opin. Colloid Interface Sci., 2007, 12, 221-231.

5 M. A. Rogers, Food Res. Int., 2009, 42, 747-753.

6 P. Wassell, G. Bonwick, C. J. Smith, E. Almiron-Roig and N. W. G. Young, Int. J. Food Sci. Technol., 2010, 45, 642-655.

7 A. G. Marangoni, N. Garti, Eds, Edible oleogels: Structure and health implications, AOCS press, Urbana, Ill, USA, (2011).

8 L. S. K. Dassanayake, D. R. Kodali and S. Ueno, Curr. Opin. Colloid Interface Sci., 2011, 16, 432-439.

9 J. Daniel and R. Rajasekharan, J. Am. Oil Chem. Soc., 2003, 80, 417-421.

10 F. G. Gandolfo, A. Bot and E. Flöter, J. Am. Oil Chem. Soc., 2004, 81, 1-6.

11 L. S. K. Dassanayake, D. R. Kodali, S. Ueno and K. Sato, J. Am. Oil Chem. Soc., 2009, 86, $1163-1173$.

12 M. A. Rogers, A. J. Wright and A. G. Marangoni, Soft Matter, 2009, 5, 1594-1596.

13 J. F. Toro-Vazquez, J. A. Morales-Rueda, E. Dibildox-Alvarado, M. Charó-Alonso, M. González-Chávez and M. M. Alonzo-Macias, J. Am. Oil Chem. Soc., 2007, 84, 9891000.

14 T. Laredo, S. Barbut and A. G. Marangoni, Soft Matter, 2011, 7, 2734-2743.

15 D. W. de Bruijne, A. Bot, in: Food Texture: Measurement and Perception (Rosenthal A. J., Editor), Aspen, Gaithersburg, MD, USA, 1999, p. 185-227.

16 A. Bot, Y. S. J. Veldhuizen, R. den Adel and E. C. Roijers, Food Hydrocolloids, 2009, 23, $1184-1189$.

17 A. Bot, R. den Adel, C. Regkos, H. Sawalha, P. Venema and E. Flöter, Food Hydrocolloids, 2011, 25, 639-646.

18 H. Sawalha, R. den Adel, P. Venema, A. Bot, E. Flöter and E. van der Linden, J. Agric. Food Chem., 2012, 60, 3462-3470.

19 R. den Adel, P. C. M. Heussen and A. Bot, J. Phys.: Conf. Ser., 2010, 247, 012025.

20 A. Lopez-Rubio and E. P. Gilbert, Trends Food Sci. Technol., 2009, 20, 576-586.

21 A. Bot, E. Flöter, in: Edible oleogels: Structure and health implications (Marangoni A G, Garti N, Editors), AOCS press, Urbana, Ill, USA, chapter 3, pp. $49-79$ (2011). 
22 H. B. Stuhrmann, N. Burkhardt, G. Dietrich, R. Junemann, W. Meerwinck, M. Schmitt, J. Wadzack, R. Willumeit, J. Zhao and K. H. Nierhaus, Nucl. Instrum. Methods Phys. Res., Sect. A, 1995, A356, 124-132.

23 E. P. Gilbert, J. C. Schulz and T. J. Noakes, Phys. B, 2006, 385-386, 1180-1182.

24 S. R. J. Kline, J. Appl. Crystallogr., 2006, 39, 895-900.

25 T. Narayanan, O. Diat and P. Bösecke, Nucl. Instrum. Methods Phys. Res., Sect. A, 2001, 467, 1005-1009.

26 L. Hernqvist, Fette, Seifen, Anstrichm., 1984, 86, 297-300.

27 B. M. Craven, in: Handbook of lipid research, volume 4, The physical chemistry of lipids from alkanes to phospholipds (Hanahan D. J. and Small D. M., Editors), p. 149-182 (1986).

28 G. Argay, A. Kálmán, S. Vladimirov, D. Zivanov-Stakic and B. Ribár, Z. Kristallogr., 1996, 211, 725-727.

29 J. M. Deutch, Macromolecules, 1981, 14, 1826-1827.

30 M. A. Rogers, A. Bot, R. S. H. Lam, T. Pedersen and T. May, J. Phys. Chem. A, 2010, 114, 8278-8285.

31 H. Sawalha, P. Venema, A. Bot, E. Flöter and E. van der Linden, Food Biophys., 2011, 6, $20-25$ 